\title{
Galaxy Formation from the Primordial Black Holes
}

\author{
Masahiro Morikawa ${ }^{a}$
}

${ }^{1}$ Department of Physics, Ochanomizu University, 2-1-1 Otsuka, Bunkyo, Tokyo 112-8610, Japan.

\begin{abstract}
Supermassive black hole (SMBH) of size $M_{B H}=10^{6-10} M_{\odot}$ is common in the Universe and it defines the center of the galaxy. A galaxy and the SMBH are generally thought to have co-evolved. However, the SMBH cannot evolve so fast as commonly observed even at redshift $z>6$. Therefore, we explore a natural hypothesis that the SMBH has been already formed mature at $z \gtrsim 10$ before stars and galaxies. The SMBH forms energetic jets and outflows which trigger massive star formation in the ambient gas. They eventually construct globular clusters and classical bulge as well as the body of elliptical galaxies. We propose simple models which implement these processes. We point out that the globular clusters and classical bulges have a common origin but are in different phases. The same is true for the elliptical and spiral galaxies. Physics behind these phase division is the runaway star formation process with strong feedback to SMBH. This is similar to the forest-fire model that displays self-organized criticality.
\end{abstract}

\section{Introduction}

Almost all the galaxy harbors a supermassive black hole (SMBH) of mass $M_{B H}=10^{6-10} M_{\odot}$ in its center[1], where $M_{\odot} \approx 2 \times 10^{30} \mathrm{~kg}$ is the solar mass. The masses of these SMBH are observed to have firm correlations with the basic components of the galaxy. For example in the case of classical bulge $(\mathrm{CB})$, its mass $M_{C B}$ has the relation $M_{B H} \approx 10^{-3} M_{C B}[3]^{1}$. In the case of globular clusters (GC), the number of them $N_{G C}$ in a galaxy has the relation $M_{B H} \approx 10^{5.5} N_{G C} M_{\odot}[4]$. Therefore it might be natural to think that the galaxy and its SMBH have co-evolved interacting with each other.

However, the standard coagulation of the stellar size black holes to form SMBH within a limited time scale turns out to be very difficult [5]. This difficulty is enhanced by the recent observations [6][7] that report many mature SMBH exist at around $z \approx 6$ (only $7.4 \times$ $10^{8}$ years after Big Bang). Various attempts to form these SMBH through the co-evolution with galaxies seem to be unnatural [8], without assuming seed black holes of huge mass $10^{6} M_{\odot}$. Moreover there seems to be an evidence that the above black hole/bulge mass relation $M_{B H} \approx 10^{-3} M_{C B}$ has already been established or even the coefficient increases toward the past[9]. All of these facts strongly suggest that the SMBH are primordial.

\footnotetext{
ahiro@phys.ocha.ac.jp

${ }^{1}$ We do not consider the similar structure called pseudo-bulge which is more like disks of spiral galaxies[2].
} 
Therefore in this paper, we start from the hypothesis that mature SMBH have been formed at around $z \approx 10$ although the mass may somewhat increase by the later accretion. Then the formation of all the structures, i.e. the stars, globular clusters, and bulges, should be attributed to the SMBH. These structures will quickly feed back to the SMBH. Thus the galaxy-SMBH co-evolution would have taken place in the early stage and several correlations above [3][4] would have already been established.

The early formation of SMBH at around $z>10$ has been studied in the model that the Bose-Einstein condensation of the self-interacting boson fields forms the dark energy [10$13]^{2}$. In this model, the unstable uniform condensation of the field can collapse to form SMBH everywhere in the Universe. This is possible because the coherent condensation does not have velocity dispersion which prevents the collapse. Furthermore in this model, the noncondensate component of the boson gas can contribute as the thermal dark matter around the SMBH.

The gravitational potential of this dark matter attracts baryon gas, and the SMBH will form a strong jet beyond the present size of the galaxy. This is plausible from the many observations at present although the jet ejection mechanism is not at all clear so far[15]. This jet will compress the surrounding gas and trigger massive star formation there[16][17]. These first stars will build globular clusters (GC), the classical bulges (CB) in spiral galaxies (SG), and the main body of the elliptical galaxies (EG). This physics is closely related with the mechanism in [18] which displays the correlation between the SMBH mass $M_{B H}$ and the velocity dispersion $\sigma$ of the stars in CB. This scenario is briefly explained in section II.

According to the above scenario, the stars of GC and CB in the spiral galaxies, would have the same origin and are indistinguishable with each other. On the other hand, their clustering features are different; GC are extended in halo and $\mathrm{CB}$ forms the core of the galaxy. This divide may come from the two distinguished flows of gas in the primordial galaxy. We clarify these flows and the origin of distinct clustering features in section III.

Furthermore an apparent similarity of CB (in SG) and EG suggests that the EG and SG have the common origin. The fact that SG is smaller than EG, in average, suggests that the strength of the jet from SMBH or the mass of SMBH are thought to be the major factor to distinguish SG and EG. We will find much interesting parameter which clearly distinguish SG and EG in our model described in section IV.

We try to find the simplest model extracting the most relevant physics from very complicated galaxy formation processes. Limitations and prospects of our approach are described in the final section $\mathrm{V}$.

\section{Early formation of supermassive black holes}

We briefly examine a possible origin of the primordial SMBH which was formed first before any other components of the galaxy. It is clear that the coagulation of the stellar size black holes to form SMBH takes too long time, more than the dynamical relaxation time scale $\tau_{r e l}=\sigma^{3} /\left(G^{2} m \rho \ln N\right)$, which turns out to be $2 \times 10^{14}$ years, where $\sigma, m, \rho, N, G$ are the velocity dispersion, mass, mass density, number of the black holes, and the gravitational constant, respectively. To bring this time scale within the cosmic age $1.38 \times 10^{10}$ years, we need seed black holes of mass $10^{5} M_{\odot}$ to start with. Furthermore dark matter gas is hopeless to collapse into SMBH because of its velocity dispersion and the angular momentum. The

\footnotetext{
${ }^{2}$ The existence of the mature SMBH before the recombination is impossible; accretion effects on the CMB significantly distort its spectrum beyond the observational limit[14].
} 
only possibility will be the collapse of dark energy $[10,11]$. This is possible if the dark energy is the Bose-Einstein condensation of fields (mass $m$ ) with an attractive self-interaction $(\lambda<0$ ). The condensate is characterized by the classical scalar field $\Psi(t, r)$ in the metric, assuming spherical symmetry,

$$
d s^{2}=\alpha^{2} \mathrm{dt}^{2}-a^{2} \mathrm{dr}^{2}-r^{2} \mathrm{~d} \theta^{2}-r^{2} \sin \theta^{2} \mathrm{~d} \phi^{2}
$$

and obeys the equation of motion,

$$
\begin{aligned}
& r a \Psi^{\prime} \dot{\Psi}-2 \dot{a}=0, \\
& -2 a^{\prime} \alpha^{2}-2 a \alpha \alpha^{\prime}+r a \alpha^{2} \Psi^{\prime 2}+r a^{3} \dot{\Psi}^{2}=0, \\
& \frac{2 a\left(r \alpha^{\prime}+\alpha\right)-2 r a^{\prime} \alpha}{r^{2} a^{3} \alpha}-2 m^{2} \Psi^{2}+\lambda \Psi^{4}-\frac{2}{r^{2}}=0, \\
& r\left(\begin{array}{c}
\dot{a} a^{2} \alpha \dot{\Psi}+a^{\prime} \alpha^{3} \Psi^{\prime} \\
+a^{3}\left(2 \alpha^{3} \Psi\left(m^{2}-\lambda \Psi^{2}\right)-\dot{\alpha} \dot{\Psi}+\alpha \ddot{\Psi}\right)
\end{array}\right) \\
& =a \alpha^{2}\left(\left(r \alpha^{\prime}+2 \alpha\right) \Psi^{\prime}+r \alpha \Psi^{\prime \prime}\right) \text {. }
\end{aligned}
$$

This set of equations easily forms black hole even if mass-less non-interacting case, which was often used to analyze critical behavior in the black hole formation process[19][20]. It was concluded that the resultant black hole mass shows scaling properties. On the other hand, more realistic bound for the black hole formation comes from the quantum fluctuations. However, it turns out that even the quantum fluctuations cannot prevent the collapse of the condensation to black hole if the mass of the boson field exceeds the Kaup limiting mass [21]

$$
M_{\text {kaup }}=0.633 \frac{\hbar c}{G m} \approx \frac{m_{p l}^{2}}{m},
$$

where $m_{p l}$ is the Planck mass. If the boson mass is the order of the present dark energy $0.01 \mathrm{eV}$, then the limiting mass becomes $1.7 \times 10^{22} \mathrm{~kg}$, almost the mass of the planet Pluto.

After the adiabatic collapse of the dark energy, some portion of the condensation becomes a black hole [19] and some other portion will melt to form thermal boson gas around the black hole[10]. This latter melting process of the condensation depends on many complex conditions and has not yet been clarified. However it would be natural to suppose that it settles down to the thermal equilibrium of mass density $\rho(r)=\rho_{0}\left(r_{0} / r\right)^{2}$ where $r$ is the distance from the central SMBH and $\rho_{0}, r_{0}$ are constants. Then this thermal uncondensed gas behaves as dark matter $[10,11]$ since it yields the commonly observed flat rotation curve.

Suppose that the SMBH thus formed at $z \approx 10$ is already surrounded by the thermal gas of dark matter with well developed gravitational potential. Then baryons are attracted within the free fall time scale about $5 \times 10^{7}$ years. At the same time the SMBH would yield energetic jets, which will trigger massive star formation along it through the ram pressure of the bow shock[16][17]. However the effect of the jet for star formation is not yet fully understood as well. If the jet conveys the momentum to the ambient baryon gas, then the pressure compresses the gas to promote the star formation. On the other hand, if the jet conveys the energy to the gas, then the heat makes the gas expand to prevent the star formation. We assume the former, the jet promotes the star formation. This assumption is partially supported by the following argument.

All the above arguments are for a steady jet with fixed direction. If the jet changes its direction rapidly less than the free fall time scale, as we will argue in the next section, then the trajectory envelope of the jet will form a superposed shock wave shells. A similar argument 
appears in [18] which displays the formation of the correlation between the mass $M_{B H}$ of the SMBH and the velocity dispersion $\sigma$ in galactic bulge. If the typical outflow radiation balances in momentum with the ambient gas of size $R$, then we have $G M_{b} M_{t o t} / R^{2}=L_{E d d} / c$, where $M_{b}=f M_{t o t}$ is the baryon mass of the galaxy, i.e. the fraction $f$ of the total mass $M_{\text {tot }}$. $L_{E d d}=4 \pi G M_{B H} c / \kappa$ is the Eddington limiting luminosity where $\kappa$ is the electron scattering opacity. Then using the virial equilibrium relation $\sigma^{2}=G M_{t o t} / R$, we have [18],

$$
M_{B H}=\frac{f \kappa \sigma^{4}}{4 \pi G^{2}} \propto \sigma^{4}
$$

This successfully describes the observations without any free parameters[3]. On the other hand if we supposed energy balance, we have an extra factor $(\sigma / c)$ on the right hand side of Eq.(4), and $M_{B H} \propto \sigma^{5}$. This predicts too small mass of SMBH and conflicts with observations. Thus the observed $M_{B H}-\sigma$ relation is consistent with the momentum balance, which suggests the jet/outflow-induced star formation.

\section{Separation into globular clusters and ellipsoids}

Now we examine how SMBH triggers star formation and makes basic components of galaxy. The first stars, as well as their direct descendants formed after the first supernova explosions, are thought to be the main ingredients of the galaxy components: classical bulge (CB), globular clusters (GC) and the elliptical galaxies (EG). These components must be formed at the same time and same mechanism since observations indicate that all of them are composed from very old population-II stars ${ }^{3}$. Then how these separations into components are processed?

We first consider the separation into GC and ellipsoids (i.e. the CB and the main body of EG). We consider that there had been two different kinds of gas velocity fields. One is caused by the local gravitational potential, and the other caused by the global cosmic turbulent flow $[22]^{4}$. The former velocity is given by the virial equilibrium and becomes a constant $v_{\text {in }} \equiv\left(4 \pi G r_{0}^{2} \rho_{0}\right)^{1 / 2}$. This is implied by the previous isothermal distribution of dark matter density $\rho=\rho_{0}\left(r_{0} / r\right)^{2}$. The latter is the scaling velocity $v_{\text {out }} \equiv(\epsilon r)^{1 / 3}$, where $\epsilon$ is a constant. This is derived by the scaling property of the cosmic turbulence[22]. This cosmic turbulence is naturally expected from the equation for the self-gravitating fluid, whose Fourier transform

$$
\frac{d v_{\vec{k}}^{\alpha}}{d t}=-i k_{\beta} \sum_{\vec{p}+\vec{q}=\vec{k}}\left(\begin{array}{c}
\left(\delta_{\alpha \gamma}-\frac{k_{\alpha} k_{\gamma}}{k^{2}}\right) v_{\vec{p}}^{\beta} v_{\vec{q}}^{\gamma}-\nu k^{2} v_{\vec{k}}^{\alpha} \\
+i \frac{k^{\alpha}}{k^{2}} 4 \pi G \delta_{\vec{k}}
\end{array}\right)
$$

is similar to the Smoluchowski coagulation equation. The scaling relation derived from this equation is applied to various observations such as the scale dependent mass density, $L / M$ ratio, magnetic field distributions, etc. They all consistently point the value $\epsilon \approx 0.3 \mathrm{~cm}^{2} / \mathrm{sec}^{3}[22]$. The former virial velocity dominates for $r<r_{*}$ and the latter turbulent velocity dominates for $r>r_{*}$, where $r_{*} \approx 8.6 \mathrm{kpc}$ if we assume $v_{\text {in }}=200 \mathrm{~km} / \mathrm{sec}$, as shown in Fig. 1.

\footnotetext{
${ }^{3}$ Some GC are reported to have several newer generation stars which might be triggered by merger for example. Here we concentrate on the main oldest generation of GC.

${ }^{4}$ This turbulence might be caused by the collapse of the condensation when SMBH are formed everywhere in the Universe or triggered by the tidal torque effect.
} 


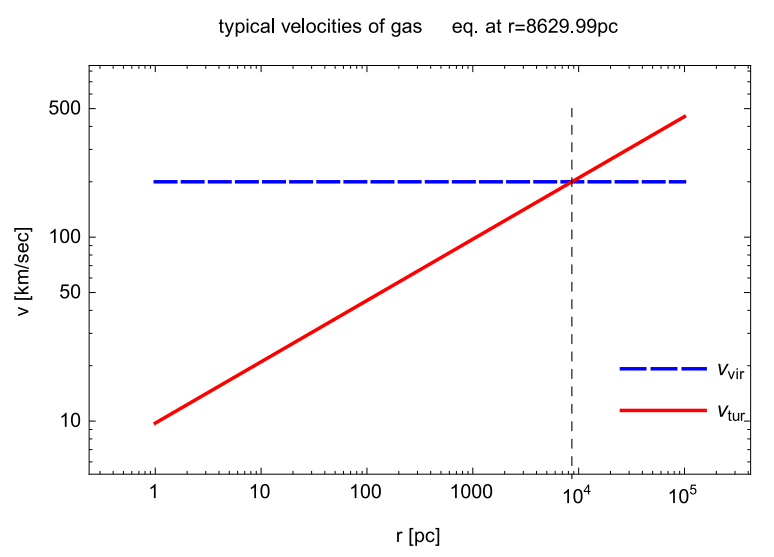

Figure 1. Velocity dispersion of ambient gas as a function of the distance $r$ from the central SMBH. There are two distinct velocity fields: The constant virial velocity field $v_{i n}=200 \mathrm{~km} / \mathrm{sec}$ (broken blue, dominates inward) and the increasing turbulent velocity field $v_{\text {out }} \equiv(\epsilon r)^{1 / 3}$ (solid red, dominates outward). They are equal with each other at $r_{*} \approx 8.6 \mathrm{kpc}$. A star formed at $r<r_{*}$ falls down toward the SMBH and forms a bulge, while the star at $r>r_{*}$ stays far from the SMBH and forms globular clusters.

The baryon gas is exerted the ram pressure from the jet and forms stars. The stars in the near region $r<r_{*}$ falls down toward the center of the potential loosing the pressure balance and suffered friction. Eventually they will form a big cluster that we call classical bulge (CB). Supernova explosions and subsequent star formation may continue. On the other hand the stars formed from the gas in the far region $r>r_{*}$ have higher speed beyond the virial equilibrium and have less probability to fall. Therefore these stars remain far from the center possibly forming small clusters that we call globular clusters (GC).

There are some specific features of the above scenario which may be significant in comparison with observations: (a) All the stars in GC and CB have the same age and chemical components because they are formed by the the same gas triggered by the common jet at the same time. (b) Each GC has almost no local angular momentum. This is because each GC is formed in the finite region where the relative gas speed is small and coherent according to the relation $v_{\text {out }} \equiv(\epsilon r)^{1 / 3}$ (Fig.1). Moreover from the same reason, once the stars are formed, they experience the cold collapse to form tight-binding cluster which has the very similar profile with each other. (c) GC are loosely bounded to the galaxy since they tend to have higher velocities $v_{\text {out }}$ than the virial equilibrium $v_{\text {in }}$. Therefore there may be significant number of stray GC in between galaxies. This point should be considered in wider viewpoint including the dwarf spheroidal galaxies[23]. They may be all the same family. These properties should be checked quantitatively. In summary, CB and GC are the same species but separated into two phases by two distinct velocity fields.

\section{Separation into spiral and elliptical galaxies}

Next we consider the separation into the spiral (SG) and elliptical (EG) galaxies in our scenario of mature SMBH. Apart from the disk, the classical bulge (CB) in SG, and EG 


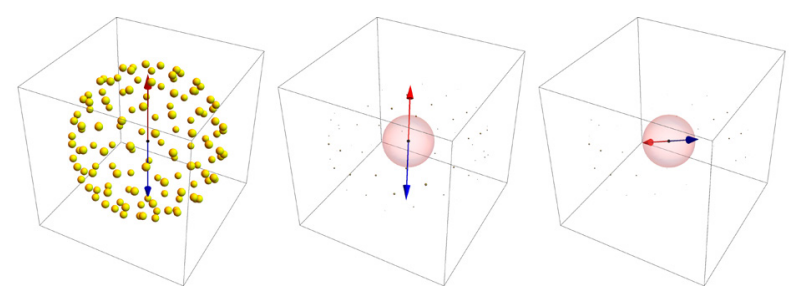

Figure 2. The galaxy formation history in case (a), high accretion rate $\lambda=1$. These are the snapshots of the gas distribution (small balls), jet (arrows), and the bulge (central pink ball) derived by the numerical calculations of Eq.6. The time flows from left to right $(t=0,150,300)$. The gas was isotropically distributed at $t=0$. Accreted objects keep exerting torque on SMBH and the jet wildly changes its direction rapidly. This rampaging jet triggers star formation in the whole solid angle. All the gas is exhausted and an elliptical galaxy is left. The parameters are $\mu=1, n=10, \kappa=$ $0.01, N=256$.

are composed from old population-II stars as globular clusters (GC). Therefore it would be natural to speculate that CB and EG are the same species but in different phases.

In order to demonstrate this process of separation, we introduce a simple model for the massive star formation by jets. Suppose the energetic jet from SMBH hits the ambient gas that is isotropically distributed around the SMBH. We assumed that the jet triggers the gas to form stars. Then those stars formed in the near region $r<r_{*}$ fall down toward the center. Some of them will give torque on the jet from SMBH through the perturbation on the accretion disk. Then the jet from SMBH changes its direction due to this torque. In the new direction of the jet, there will be plenty of fresh gas ready to form stars. Thus the jet will trigger new star formation in this rich gas environment. This yields further torque on the SMBH. This feedback dynamics will be simply represented by the following model:

$$
\begin{aligned}
& \left.\dot{g}_{i}(t)=\quad-\mu \mid \overrightarrow{[J}(t)\right]\left._{n} \cdot \overrightarrow{s_{i}}\right|^{\alpha}|\vec{J}(t)| g_{i}(t), \\
& \dot{\vec{J}}(t)=-\lambda\left[\vec{J}(t) \times \sum_{i} \vec{s}_{i} \dot{g}_{i}(t)\right]_{n} \sum_{i} \dot{g}_{i}(t)-\kappa \vec{J},
\end{aligned}
$$

where the vector $\vec{J}(t)$ represents the jet (direction=axes of the jet, amplitude $=$ strength of the jet). The scalar $g_{i}(t)$ represents the amount of gas in the $i$-th direction $\overrightarrow{s_{i}}$, where $\left.\overrightarrow{\left\{s_{i}\right.}\right\}_{1 \leqq i \leqq N}$ covers the whole solid angle. The parameter $\alpha$ simply controls the beam widths and the symbol $[*]_{n}$ represents the unit vector with the same direction $*$. The directions $\left.\overrightarrow{\left\{s_{i}\right.}\right\}_{1 \leqq i \leqq N}$ are designed based on the Fibonacci-Himawari coordinate system using the golden angle $\bar{\beta}=$ $137.5^{\circ} \ldots$ so that it covers the whole solid angle with uniform density: $\overrightarrow{s_{i}}=(s \sin \gamma, s \cos \gamma, c)$ with $c=1-2\left(i / i_{\text {total }}\right), s=\sqrt{1-c^{2}}, \gamma=\beta i$. The first line of Eq.(6) represents the gas reduction process by the star formation triggered by the jet. This is proportional to the star formation rate $\mu$, the strength of the jet toward the direction $i$ with the jet collimation parameter $\alpha$, and the amount of gas at that direction $g_{i}(t)$. The second line represents the time change rate of the jet. This is proportional to the feeding efficiency $\lambda$ of the formed star to the SMBH, and the torque exerted by the falling stars just formed. A natural fade-out term parameterized by $\kappa$ is added.

There are two typical cases in this model(Figs.2, 3). (a) The jet is active and rapidly changing its direction until finally all the ambient gas is exhausted to form stars (Fig. 2). (b) The jet is active until some intermediate time when the final jet direction is fixed. There are 


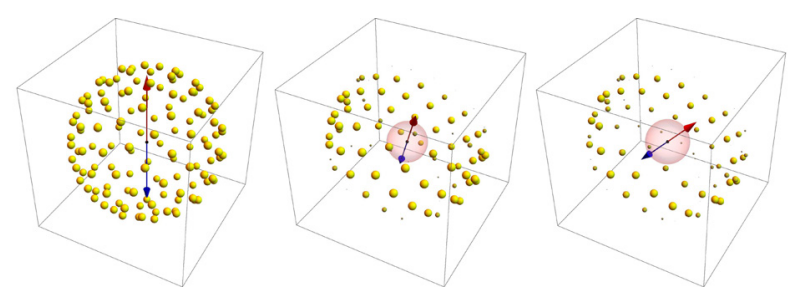

Figure 3. The galaxy formation history in case (b), low accretion rate $\lambda=0.1$. The same as Fig.2 but with low accretion rate. The jet is inactive and its direction does not change much. Therefore the gas is left in a torus form almost perpendicular to the final jet direction. The gas will eventually settle to form a disk and a spiral galaxy is left.
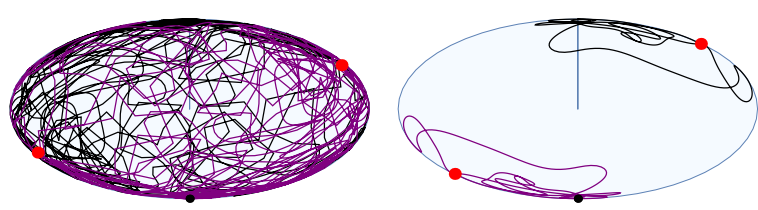

Figure 4. Trajectories of the moving jet-pair direction in the Mollweide projection of the whole solid angles; horizontal and vertical axes respectively represent the longitude and latitude. Small black and big red point pairs represent the initial and final jet directions, respectively. (left) The jet trajectory for the case with large accretion rate $(\lambda=1)$. The jet runs violently in the whole solid angle. This is the case of elliptical galaxy. (right) The same but small accretion rate $(\lambda=0.055)$. The jet runs gentle and eventually the direction is settled. This is the case of spiral galaxy.

finite remaining gas that failed to form stars and distributed almost perpendicular direction to the jet (Fig. 3) ${ }^{5}$. In the former case (a), the resultant structure has almost no gas but has a big star cluster at the center and small clusters GC around it, both composed from the stars of the same age. This is the typical elliptical galaxy. On the other hand in the latter case (b), the structure is the central star cluster bulge and GC, which are surrounded by the remaining gas. This gas would eventually form disk structure, in which new stars are going to be formed spontaneously. This is the typical spiral galaxy. Thus the main parts of the elliptical and spiral galaxies born at the same time by the same mechanism. Only the degree of jet activity separates them into the two phases SG and EG. The jet direction trajectories in both cases are compared in Fig.4.

The distinction of the two phases seems to be sharp if parameterized by the accretion rate $\lambda$, but not by the other parameters, as shown in Fig.5. If $\lambda$ is large, then the torque exerted from the falling stars strongly changes the jet direction so that more star formation takes place in the fresh ambient gas. This further exert strong torque and this runaway continues until all the gas is exhausted. On the other hand if $\lambda$ is small, then the falling stars exert only weak torque to change the jet direction. Then the jet cannot hit the sufficient amount of fresh gas to yield torque and eventually the jet direction is settled, leaving the torus shape

\footnotetext{
${ }^{5}$ If the settled jet-pair happens to point toward the gas remaining regions, then the small scale star formation activity may be still triggered which further induce density wave arm structure emanating from there.
} 


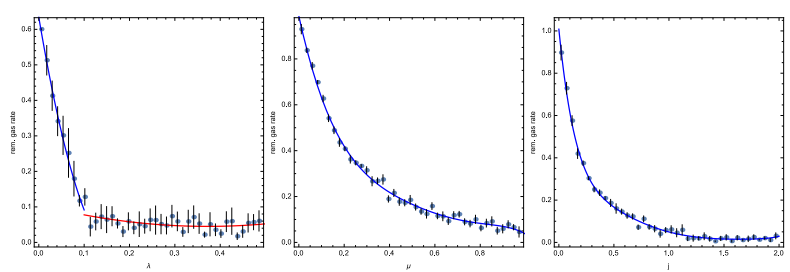

Figure 5. The fraction of the remaining gas that didn't form stars at $t=300$. Each parameter is changed from the standard set $\lambda=1, \mu=1, j=1$. (left) The case the accretion rate $\lambda$ is changed. The remaining gas fraction has a sharp bend only in this case. (center) The case the star formation rate $\mu$ is changed. (right) The initial jet strength $j$ is changed. If the jet activity does not exist at all $(j=0)$, it may yield a bulge-less spiral galaxy. The other parameters are the same as in Fig. 2.

gas distribution around the central bulge. The positive feedback eventually stops, and no runaway takes place.

This galaxy formation process has similar physics to the forest-fire model in complex systems[24, 25]. This model is composed of many trees on a two-dimensional lattice. Each tree on a lattice site has a finite probability $p$ to ignite spontaneously. If ignite, the fire spreads to the neighboring trees with some probability. On the site of the burnt tree, a new tree has a chance to grow with some probability. The main feature is that the fire-fire correlation length $\xi(p)$, depends on $p$, determines the asymptotic two distinct states: (a) The fire dies out if $\xi(p)$ exceed the system size $L$. (b) The fire is sustained if $\xi(p)<L$. Percolation caused by the positive feed back or runaway process is the common feature in our galaxy model and the forest-fire model although the former is deterministic, as Eq.(6), and the latter is probabilistic.

The parameter of accretion rate $\lambda$ may particularly be important to divide the galaxies into elliptical and spiral (Fig.5 left). The special value $\lambda_{*} \approx 0.1$ divides the EG $\left(\lambda>\lambda_{*}\right)$ and $\operatorname{SG}\left(\lambda<\lambda_{*}\right)$. The marginal case $\lambda \approx \lambda_{*}$ may correspond to the S0 galaxies. If the system has larger angular momentum in total, then the falling stars have less chance to fall near SMBH and therefore yield smaller accretion rate $\lambda$. Then this system tends to become a spiral galaxy. Moreover in this spiral galaxy case, the central cluster bulge is generally smaller than the elliptical galaxy provided the initial amount of ambient gas were the same with each other.

Other parameters are also important to divide the phases and yield variety of galaxies. The star formation rate $\mu$ would be higher in the denser environment of gas such as in the bottom of the gravitational potential. Therefore EG have much chance to be formed in the cluster and SG in the field.

Any relic of the above star-forming jet activity may be observed in the very early galaxies at distance. Most of the early stage spiral galaxies are generally thought to have very irregular shape as the rampaging jet which randomly forms stars and the remaining gas is not in ordered distribution. Thus, early irregular galaxies evolves into SG later. However this irregularity of early galaxies may be contaminated by the frequent collisions of galaxies in the dense environment in the past.

The jet activity may leave its trace in the faint structures in a spiral galaxy. The final stage of the jet would be gentle and the jet will generally have small precession. This jet may form a double-cone shape region in which the stars are massively formed. Subsequent supernova 
explosions of those stars may leave high energy electrons and protons there captured and stored in the magnetic fields. This relic ionized region will be observed in the early galaxies. However this relic structure may be contaminated with the relatively recent jet activity which often observed[26].

\section{5 conclusions and prospects}

We proposed a scenario that the primordial supermassive black hole (SMBH) made the galaxy. Energetic jets from the SMBH give ram pressure to the ambient gas to trigger the massive star formation. We focused on the following two aspects of this scenario.

The first aspect is the comparison of a globular cluster (GC) and a classical bulge (CB). According to our scenario, they are the same species but in distinct phases. We proposed that the difference comes from two kinds of velocity fields of the gas around the SMBH: (a) the virial velocity field associated with the dark matter distribution that dominates inside of the galaxy, and (b) the cosmic turbulent field, which obeys the Kolmogorov scaling, that dominates outside. Some portion of the star formed by the jet in (a) falls into the center and yields CB. On the other hand the star formed in (b) stays in halo and yields GC. Thus the two species $\mathrm{CB}$ and $\mathrm{GC}$ are formed simultaneously from the same gas, but in distinct velocity fields.

The second aspect is the comparison of the spiral galaxies (SG) and the elliptical galaxies (EG). According to our scenario, CB (in SG) and EG are the same species but in distinct phases. We proposed that the difference comes from the amount of jet activity. We introduced a simple model that describes this discrimination. According to this, (a) large feeding efficiency $\lambda$ makes strong torque on SMBH that causes runaway flipping of the jet direction in the whole solid angle. Finally all the gas is exhausted and an EG is left. On the other hand (b) small $\lambda$ makes weak torque and the jet direction change mildly without runaway. This makes smaller cluster in the center (CB) and the leftover gas will eventually form a disk, leaving a SG. Thus the existence/absence of the runaway separates the same species into two phases. This process has similar physics to the forest-fire model in complex systems.

Thus a SMBH defines the center of a galaxy and various star clusters in the galaxy (GC, CB, EG) are nurtured by the SMBH through the energetic jet emanating from the SMBH. According to this scenario, the hypothesis of the population-III stars that formed spontaneously may not be necessary.

Our simple analysis may be a useful supplement to the solid simulations of galaxy formation including all physical processes. We ignored many relevant dynamics such as major/minor mergers, detail of the star formation process, back reaction to the jet, jet collimation,... We would like to check whether the present model does include the most relevant process among them and refine our model through further study.

The author would like to thank Hideaki Mouri, Tohru Tashiro and all the laboratory members for fruitful discussions.

\section{References}

[1] J. Kormendy and D. Richstone, Annu. Rev. Astron. Astrophys. 33, 581 (1995).

[2] J. Kormendy, arXiv:1504.03330v1 (2015).

[3] K. Gultekin et al. ApJ 698, 198 (2009). 
[4] G. Harris and W. Harris, MNRAS 410, 2347H (2011).

[5] M. Rees, Annu. Rev. Astron. Astrophys. 22, 471 (1984).

[6] P. Marziani and J. Sulentic, New Astron. Rev. 56, 49 (2012).

[7] X. Wu et al., Nature 518, 512 (2015).

[8] M. Latif et al., arxiv1404.5773v2 (2014).

[9] A. Schulze \& L. Wisotzki, MNRAS 438, 3422 (2014).

[10] M. Nishiyama, M. Morita, and M. Morikawa, arXiv:astro-ph/0403571 (2004).

[11] T. Fukuyama and M. Morikawa, Prog. Theor. Phys. 115, 1047 (2006).

[12] T. Fukuyama, T. Tatekawa and M. Morikawa, JCAP06, 033 (2008).

[13] T. Fukuyama and M. Morikawa, Phys. Rev. D80, 063520 (2009).

[14] B.J. Carr, K. Kohri, Y. Sendouda, J. Yokoyama, Phys.Rev.D81, 104019 (2010).

[15] The Formation and Disruption of Black Hole Jets, ed. by I. Contopoulos, D. Gabuzda, and N. Kylafis, Astrophysics and Space Science Library 414 (2015).

[16] A. Reines and A. Deller, ApJ 750, L24R (2012).

[17] C. Liu, Z. Gan, and F. Xie, Research in Astronomy and Astrophysics 13, 899 (2013).

[18] A. King, ApJ 59, L27 (2003).

[19] M. Choptuik, Phys. Rev. Lett. 70, 9 (1993)

[20] C. Gundlach, Living Rev. Relativity 10, 5 (2007).

[21] D. Kaup, Phys. Rev. 1721331 (1968).

[22] A. Nakamichi and M. Morikawa, JCAP01, 011 (2010).

[23] S. van den Bergh, Monthly Notices of the Royal Astronomical Society 385, L20 (2007).

[24] C. Henley, Bull. Am. Phys. Soc. 34, 838 (1989).

[25] P. Bak, and C. Tang, Phys. Lett. A 147, 297 (1990).

[26] G. Dobler et al., ApJ 717, 825 (2010). 